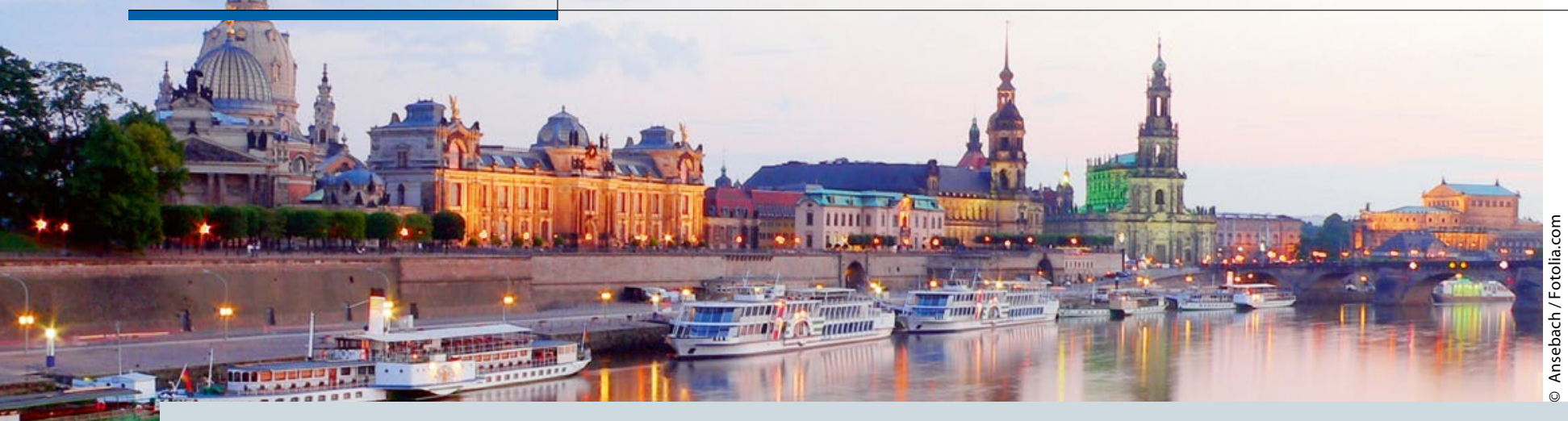

47. Jahrestagung der Deutschen Dermatologischen Gesellschaft, Dresden Dermatologie im Fluss

Anfang Mai war Dresden wieder einmal vier Tage lang die dermatologische Hauptstadt Deutschlands: Mehr als 3.100 Teilnehmer waren gekommen, um sich an der Elbe ihr fachliches Update zu holen. Auf den folgenden Seiten finden Sie ausgewählte Highlights im Häppchenformat zum Nachlesen.

\section{Stiefkind künstliche UV-Strahlung}

— Arbeitsplätze mit einer hohen künstlichen UV-Belastung sind deutlich seltener als solche mit natürlicher UV-Exposition. Etwa 300.000 Arbeitnehmer sind laut Prof. Dr. Manigé Fartasch, Universität Bochum. davon betroffen. Epidemiologisch sah der ärztliche Sachverständigenrat bisher die Korrelation von arbeitsbedingter Exposition und dem Auftreten von Malignomen noch nicht als begründet an. Es gibt zwar Höchstwerte zur Vermeidung akuter Schäden $\left(30 \mathrm{~J} / \mathrm{m}^{2}\right)$, die Relevanz dieser Werte zur Prävention von Langzeitschäden ist laut Fartasch aber fraglich. Nach ihren Untersuchungen gibt es bei Tätigkeiten wie UV-Kleben, Schweißen oder Glasbläserei spezifische Belastungen. Unter 29 Glasbläsern fand sie beispielsweise bei etwa der Hälfte regelmäßig und teils täglich auftretende Erytheme an Gesicht oder Händen. Aus medizinischer Sicht seien hier unbedingt Vorsorgeuntersuchungen von Haut und Auge sowie Unterweisungen nötig, forderte sie. Aus dermatologischer Sicht sei es sinnvoll, bei Auftreten von Hauttumoren, auch von Carcinoma in situ, bei entsprechender Berufsanamnese und atypischer Lokalisation aufmerksam zu sein. Zudem müsse bei Photodermatosen immer auch der Beruf abgefragt und an künstliche UV-Strahlung gedacht werden, riet Fartasch. Friederike Klein

Fartasch M. Arbeitsplätze mit künstlicher UVBelastung. Symposium „Berufsdermatologie“

\title{
Hautkrebs - der lange Weg zur Berufskrankheit
}

— In seiner 95. Sitzung hat der ärztliche Sachverständigenbeirat „Berufskrankheiten“ beim Bundesministerium für Arbeit und Soziales am 18. und 19. September 2012 einstimmig die wissenschaftliche Begründung für eine neue Berufskrankheit BK 5103 „Plattenepithelkarzinome oder multiple aktinische Keratosen der Haut" angenommen, berichtete Prof. Dr. Thomas L. Diepgen, Klinische Sozialmedizin der Universität Heidelberg. Dabei gelten aktinische Keratosen (AK) als multipel bei mehr als fünf einzelnen $A K$ pro Jahr oder konfluierend in einer Fläche von mehr als $4 \mathrm{~cm}^{2}$ (Feldkanzerisierung). Voraussetzungen für die Feststellung der Berufkrankheit sind die zweifelsfreie Sicherung der Diagnose, die Lokalisation der Hautveränderungen an beruflich exponierten Körperstellen und eine ausreichend intensive berufliche UV-Exposition. Die BK 5103 umfasst dabei derzeit nur beruflich

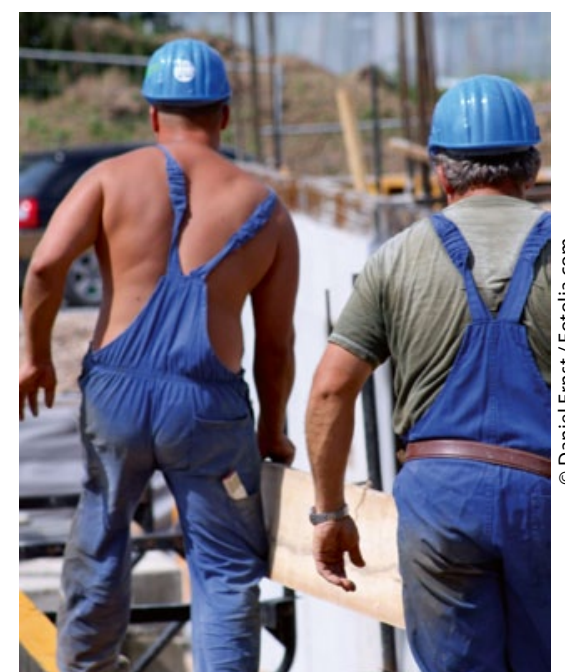

bedingte Kanzerosen durch natürliche UVQuellen, künstliche UV-Quellen müssen noch weiter untersucht werden. Auch außen vor sind Basalzellkarzinome, sie fallen derzeit nicht unter die BK 5103. Laut Diepgen ist hierzu eine Fall-Kontroll-Studie geplant, um die wissenschaftliche Basis dafür zu untermauern. Die Dermatologen in Deutschland hätten mit ihren vermehrten Anzeigen von "Quasi-Berufskrankheiten" nach § 9 Abs. 2 SGB VII wesentlich dazu beigetragen, dass die BK 5103 kommt, betonte Diepgen. Gestützt wurde die Initiative außerdem durch zahlreiche Sozialgerichtsurteile.

Im Januar versandte das Ministerium die wissenschaftlichen Begründungen zur BK 5103 an die Deutsche Dermatologische Gesellschaft und die Deutsche Gesellschaft für Arbeits- und Umweltmedizin. Der nächste Schritt ist nun die Veröffentlichung im Bundesanzeiger. Die dann notwendige Novellierung der Berufskrankheitenverordnung erwartet Diepgen allerdings erst im Frühjahr 2014 - die im Herbst anstehende Bundestagswahl bremst die Umsetzung.

Friederike Klein

Diepgen TL. Aktuelle Entwicklung zum beruflichen Hautkrebs: BK 5103? Symposium „Berufsdermatologie"

Die „ewige Baustelle“ BK 5103 scheint 2014 jetzt doch fertig zu werden: Dann wird Hautkrebs bei Outdoor-Arbeitern als Berufskrankheit anerkennungsfähig sein. 